\title{
A modified Trastuzumab antibody for the immunohistochemical detection of HER-2 overexpression in breast cancer
}

\author{
G Bussolati*,', F Montemurro' ${ }^{2}$ L Righi', M Donadio ${ }^{3}$, M Aglietta ${ }^{2}$ and A Sapino' \\ 'Department of Biomedical Sciences and Human Oncology, University of Turin, Torino, Italy; ${ }^{2}$ Unit of Medical Oncology, Institute for Cancer Research and \\ Treatment, Torino, Italy; ${ }^{3}$ Department of Medical Oncology, San Giovanni Hospital, Torino, Italy
}

\begin{abstract}
The immunohistochemical determination of HER-2 to identify patients with advanced breast cancer candidates for Trastuzumab treatment proved neither accurate nor fully reliable, possibly because none of the current reagents detects the specific antigenic site target of Trastuzumab. To circumvent this problem, we conjugated the $\mathrm{NH}_{2}$ groups of Trastuzumab with biotin, and the compound obtained, designated BiotHER, was added directly to tissue sections. Biotin-labelling was revealed with horseradish peroxidaseconjugated streptavidin. Specificity and sensitivity of BiotHER immunostaining with respect to HER-2 amplification were tested on 164 breast carcinoma samples. BiotHER staining was detected on the tumour cell membrane of I2\% of all specimens and in $49 \%$ specimens with gene amplification, while absent in nonamplified tumours. Predictivity of BiotHER status with respect to the clinical outcome was analysed in 54 patients with HER-2 amplified advanced breast cancer treated with Trastuzumab plus chemotherapy. BiotHER staining, detected in $50 \%$ of tumours with HER-2 amplification, was an independent predictor of clinical outcome. In fact, BiotHER positivity was independently associated with increased likelihood of tumour response and reduced risk of tumour progression and death. Biotinylated Trastuzumab can thus be used for immunohistochemical detection of HER-2 overexpression in breast cancer, and has the potential to identify patients likely to benefit from Trastuzumab treatment.

British Journal of Cancer (2005) 92, I26I-1267. doi:I0.1038/sj.bjc.6602507 www.bjcancer.com

(c) 2005 Cancer Research UK
\end{abstract}

Keywords: Trastuzumab; HER-2; breast carcinoma; biotin; predictivity

Overexpression of the HER-2 transmembrane receptor is detected in $25-30 \%$ of human breast cancers (Slamon et al, 1987, 1989) and is a direct result of gene amplification (Pauletti et al, 1996) in approximately $90-95 \%$ of cases. Demonstration of HER-2 antigen overexpression in breast cancer constitutes the rationale for treatment with Trastuzumab (Baselga et al, 1998). Trastuzumab consists of the antigen-binding fragment $(\mathrm{Fab})$ of the murine $\mathrm{mAb}$ 4D5, directed against the extracellular domain (ECD) of HER-2, spliced to the Fc fragment of human IgG (Baselga et al, 1998). The $4 \mathrm{D} 5 \mathrm{mAb}$ was humanised to minimise the immunogenicity associated with the murine counterpart and to maximise its potential to recruit endogenous immune effector cells (Tokuda et al, 1996; Baselga et al, 1998). As a single agent, Trastuzumab produced a response rate of $15-26 \%$ in women with HER-2overexpressing advanced breast cancer (Cobleigh et al, 1999; Vogel et al, 2002). Combined with chemotherapy, Trastuzumab has resulted in increased response rate and survival (Slamon et al, 2001, Montemurro et al, 2004b) in this clinical setting of patients.

The clinical response to Trastuzumab can be predicted by immunohistochemical (IHC) determination of HER-2 receptor expression in tumour cell membranes (Slamon et al, 2001;

* Correspondence: Dr G Bussolati, Dipartimento di Scienze Biomediche e Oncologia Umana, Università di Torino, Via Santena 7, 10126 Torino, Italy; E-mail: gianni.bussolati@unito.it

Received 8 September 2004; revised I January 2005; accepted 8 February 2005
Montemurro et al, 2004b). However, establishing the presence of HER-2 gene amplification by fluorescence in situ hybridisation (FISH) seems to be a more accurate, reliable and cost-effective method for selecting patients eligible for Trastuzumab therapy (Elkin et al, 2004) than IHC, perhaps because none of the available IHC procedures detect the Trastuzumab epitope. In fact, the Trastuzumab-binding site has been mapped to the C-terminal portion of domain IV in the juxtamembrane region of the ECD (Cho et al, 2003). On the contrary, other commercial products used for HER-2 receptor testing do not recognise the same epitope as Trastuzumab. Specifically, Herceptest, and mAb CB11 recognise the intracellular domain of HER-2 (Corbett et al, 1990; Ceccarelli et al, 1999). On the other hand, mAb TAB250 recognises the ECD but the epitope is unknown (Ceccarelli et al, 1999) (Figure 1). Previously, we demonstrated that by combining the score values obtained by CB11 and TAB $250 \mathrm{mAbs}$ in a double scoring system, it was possible to predict the gene status in $58 \%$ of cases of breast cancer (Sapino et al, 2003), thus proving the interest for IHC detection of the ECD.

However, to predict Trastuzumab activity, it is essential to verify the availability of the Trastuzumab target epitope, especially since several studies have demonstrated the occurrence of tumours expressing variants of the HER-2 protein (Christianson et al, 1998; Kwong and Hung, 1998; Molina et al, 2001, 2002). Many cell surface transmembrane proteins, including growth factor receptors, can be released from the cell surface by a general shedding system activated by several independent mechanisms. The ECD of the HER-2 may be cleaved and shed from the receptor and can be 


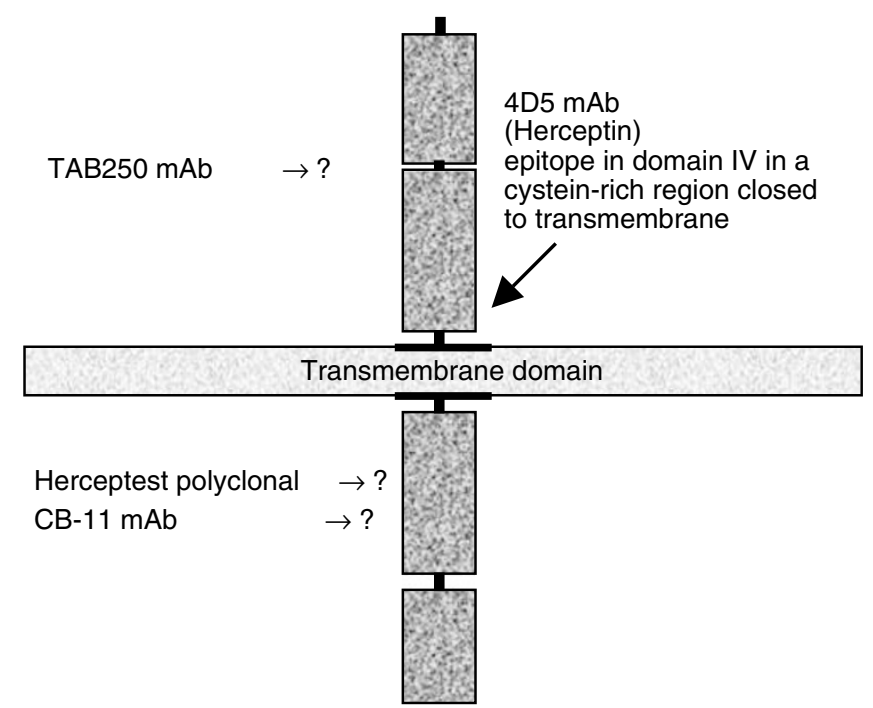

Figure I Site of binding of different antibodies used in the $\mathrm{IHC}$ detection of HER-2 overexpression in breast cancer. The exact epitope recognised by some reagents is presently unknown.

detected in serum of $35-40 \%$ patients with metastatic breast cancer as a protein of approximately $105 \mathrm{kDa}$ (Hayes et al, 2001). Such shedding process is actively regulated by proteolytic processes. In addition, in in vitro experiments using Trastuzumab as antibody for immunoprecipitation analysis, it has been shown that the soluble ECD in the medium maintains the Trastuzumab epitope, which is lost in the cell lysates (Codony-Servat et al, 1999). This shedding generates an $\mathrm{NH}_{2}$-terminally truncated HER-2 product of $M_{\mathrm{r}} 95000$ demonstrated in cell lines and in breast cancer tissues (Christianson et al, 1998; Molina et al, 2001, 2002). In these circumstances, patients are still selected as candidates for Trastuzumab therapy on the basis of gene amplification analyses and staining procedures that recognise the intracytoplasmic portion, but treatment is doomed to fail because of the lack of the Trastuzumab epitope.

A straightforward approach would be to use Trastuzumab as the primary $\mathrm{Ab}$ in IHC. However, technical reasons impede this because antigen detection using a primary $A b$ of the same species as the target tissue is complicated by high background staining. The present study demonstrates that, following modification, Trastuzumab can be used reliably to detect overexpression of its antigen by IHC. In a series of cancer samples of different organs, this novel IHC test was compared to commercial antibodies in detecting HER-2 overexpression and with in situ hybridisation procedures that reveal gene amplification. Finally, in a series of patients with HER-2-amplified advanced breast cancer treated with Trastuzumab plus chemotherapy, we retrospectively studied the predictivity of BiotHER status with respect to clinical outcome.

\section{MATERIALS AND METHODS}

\section{Rationale for the procedure and preparation of reagents}

The modification of Trastuzumab consisted in conjugating the $\mathrm{mAb}$ to biotin which binds $\mathrm{NH}_{2}$ groups of the mAb. Trastuzumab, commercially available as Herceptin ${ }^{\mathbb{R}}$ (Roche, Hertfordshire, UK), is distributed in vials for use in breast cancer treatment. One milligram of Trastuzumab from a vial of Herceptin ${ }^{\circledR}$ was diluted in saline solution $\left(1 \mathrm{mg} \mathrm{ml}^{-1}\right.$ concentration) and dialysed overnight in $0.1 \mathrm{M} \mathrm{Na} \mathrm{Na}_{2}, \mathrm{pH} 8.5$. To $1 \mathrm{ml}$ solution, $0.12 \mathrm{ml}$ of $\varepsilon-$ caproylamido-biotin- $n$-hydroxy-succinimide ester (Biospa, Mila- no, Italia) was added. The preparation was mixed by gentle agitation for $4 \mathrm{~h}$ at room temperature (RT) and dialysed in phosphate-buffered saline (PBS). The product, designated $\mathrm{Bi}$ otHER stock solution, was stored frozen at $-80^{\circ} \mathrm{C}$ in $20 \mu \mathrm{l}$ aliquots and sublimated. The lyophilized $\mathrm{mAb}$ was stored in vials.

\section{Immunohistochemical methods for BiotHER immunostaining}

To set up the IHC procedure for BiotHER, we used cell pellet of BT474 breast cancer cells (American Type Culture Collection, Manassas, VA, USA) and a tissue array of 10 breast cancers with different $H E R-2$ gene status (five amplified and five nonamplified tumours). Cells were maintained at $37^{\circ} \mathrm{C}$ and $5 \% \quad \mathrm{CO}_{2}$ in Dulbecco's modified Eagle's medium 13 (DMEM) (Sigma-Aldrich) containing $10 \%$ fetal calf serum (Biochrom-Berlin). Confluent cells were scraped and centrifuged. Cell pellets were fixed in $10 \%$ neutral-buffered formalin, then embedded in paraffin. Deparaffinised tissue sections were brought to PBS, then covered with $25 \mu \mathrm{l}$ BiotHER, final dilution $1: 2000$ in PBS (protein concentration $=0.5 \mu \mathrm{g} \mathrm{ml}^{-1}$ ). Sections were incubated for $1 \mathrm{~h}$ at $\mathrm{RT}$, washed twice for $5 \mathrm{~min}$ in PBS. Biotin label of BiotHER was then revealed incubating with horseradish peroxidase (HRP)-conjugated streptavidin diluted 1:50 (BioGenex, San Ramon, CA, USA) for $13 \mathrm{~min}$ at RT. Since preliminary tests showed that BiotHER reactivity was not improved by antigen retrieval (MW or proteinase digestion), we eventually avoided this procedure. Sections were then washed in PBS and the reaction was routinely developed in a solution of $3^{\prime}$-3-diaminobenzidine (DAB) and $\mathrm{H}_{2} \mathrm{O}_{2}$ for $5 \mathrm{~min}$. Nuclei were counterstained with Mayer Haemalum for $30 \mathrm{~s}$. Dehydration in alcohols, clarification in xylol and mounting in Entellan followed. Positivity corresponded to membrane staining. Evaluation was performed with the four-tiered scoring system (see below). For quality control analysis, conjugation of biotin to Trastuzumab was performed every week for a total of five biotinylation processes and the obtained BiotHER compound was tested at each time point by IHC in the control tissues. To test preservation of immunoreactivity, lyophilised BiotHER stock was tested every 2 weeks by IHC procedures on the control tissue array.

To correlate gene amplification and BiotHER immunoreactivity with Herceptest and TAB250 immunoreactivity, we tested these antibodies on tissue arrays prepared from tumours of different organs (164 primary breast carcinomas, 28 colon, 28 lung, 28 ovarian and 26 prostate cancers). All tissues were formalin fixed and paraffin embedded. In 24 breast carcinomas, immunohistochemistry using 4D5 mAb was also performed.

Herceptest (Dako Corp., Carpenteria, CA, USA) was utilised according to the manufacturer's instructions. TAB250 mAb (Zymed, San Francisco, CA, USA) was used as previously reported (Sapino et al, 2003). After blocking nonspecific binding sites (Histostain Plus kit, Zymed), sections were incubated for $30 \mathrm{~min}$ at $37^{\circ} \mathrm{C}$ with $\mathrm{TAB} 250$ diluted $1: 40$, then incubated for $20 \mathrm{~min}$ with secondary biotinylated anti-mouse Ig antibody ( $1: 50$, BioGenex), followed by HRP-conjugated streptavidin (1:50, StrAviGen MultiLink Kit, BioGenex) for additional $20 \mathrm{~min}$ at RT. Sections were also stained using the 4D5 mAb (not commercially available and kindly supplied by Dr PG Natali, Regina Elena Cancer Institute, Rome, Italy). For IHC, mAb 4D5 was diluted 1:1000 following the methods used for TAB250 mAb. As with BiotHER, we did not observe any advantages for reactivity of $4 \mathrm{D} 5$ by antigen retrieval procedures. The four-tiered scoring system $(0,1+, 2+$ and $3+$ score values) suggested by the manufacturers to evaluate the results of Herceptest and TAB250 in breast cancer tissue was applied to these Abs and also to BiotHER and 4D5 results. HER-2 protein expression was defined as negative (scores 0 and $1+$ ) or positive (scores $2+$ and $3+$ ). This analysis was performed double-blindly. 
All cases were then studied with Chromogenic Insitu Hybridization (CISH) and/or FISH to assess HER-2 gene amplification. BiotHER sensitivity and specificity were calculated using the known HER-2 gene status of the tumour as the gold standard.

\section{Fluorescence in situ hybridisation or CISH procedures}

PathVysion HER-2/neu probe kit (Vysis Inc., Downers Grove, IL, USA) was used for FISH analysis. In brief, sections were baked overnight at $56^{\circ} \mathrm{C}$, and invasive carcinoma components were selected based on haematoxylin and eosin-stained sections, deparaffinised in CitriSolv, dehydrated in $100 \%$ ethanol and airdried. Slides were then treated with proteases for 45-60 min, denatured and hybridised overnight at $37^{\circ} \mathrm{C}$ with the probes (HER2/neu/CEP17 SG probe 35-171060, Vysis Inc., Downers Grove, IL, USA). Slides were washed with posthybridisation buffer at $72^{\circ} \mathrm{C}$, counterstained with $4^{\prime}, 6^{\prime}$-diamidino-2-phenylindole (DAPI), mounted and stored in the dark prior to signal enumeration. Slides were first scanned at low power with a DAPI filter to identify areas of optimal tissue digestion and nonoverlapping nuclei. Cases were scored as amplified when the ratio of HER-2/chromosome 17 signals were $\geqslant 2.0$. This analysis was performed double-blindly. For CISH analysis, the sections were deparaffinised in xylene and $99 \%$ alcohol, air-dried for $10 \mathrm{~min}$ and then heated above $98^{\circ} \mathrm{C}$ for 15 min in CISH Tissue Heat Pretreatment. The sections were then rinsed in $\mathrm{dH}_{2} \mathrm{O}$ and digested with pepsin for $10 \mathrm{~min}$. Afterwards, sections were washed in $\mathrm{dH}_{2} \mathrm{O}$, dehydrated with graded alcohol, and air-dried. The ready-to-use digoxigenin-labelled HER-2/neu probe (consisting of two contig BAC clones; Zymed Lab) was applied onto slides, which were covered by $14 \times 14 \mathrm{~mm}$ coverslips (10 $\mu \mathrm{l}$ probe mixture/slide). The slides were denatured on a hot plate $\left(94-95^{\circ} \mathrm{C}\right)$ for $5 \mathrm{~min}$, and the hybridisation was performed overnight at $37^{\circ} \mathrm{C}$. After hybridisation, the slides were washed with $0.5 \times$ SCC prewarmed at $75^{\circ} \mathrm{C}$ for $5 \mathrm{~min}$, followed by three washes in $\mathrm{dH}_{2} \mathrm{O}$. The HER-2/neu probe was detected with sequential incubations with mouse anti-digoxigenin antibody for $45 \mathrm{~min}$ followed by incubation with polymerised HRP-anti-mouse antibody for another $45 \mathrm{~min}$ and diaminobenzidine according to the manufacturer's instructions (Zymed). Tissue sections were lightly counterstained with methyl green.

Amplified cases had both low level amplification (showing 6-10 signals per nucleus in $>50 \%$ of cancer cells, or a small gene copy cluster), and high level HER-2 gene amplification (showing a large gene copy cluster in $>50 \%$ of carcinoma cells or $>10$ separate gene copies), as defined in the original report (Sapino et al, 2003).

\section{Patients}

To evaluate the impact of BiotHER immunostaining on Trastuzumab response, IHC analysis with BiotHER was performed in specimens from 54 women with HER-2 amplified advanced breast cancer. These cases were selected because: (i) they had been treated with Trastuzumab combined with chemotherapy, (ii) their tumour blocks were available for retesting and (iii) their follow-up data was available. All 54 cases were re-evaluated with BiotHER and FISH. The patients had started treatment between September 1999 and July 2004. Immunohistochemical positivity was originally scored $3+$ in 45 patients and $2+$ in nine patients. For seven of these $2+$ tumours, a FISH test showing HER-2 amplification had been obtained before initiating therapy with Trastuzumab.

Treatment In all 54 patients, Trastuzumab was administered using the weekly schedule $\left(4 \mathrm{mg} \mathrm{kg}^{-1}\right.$ loading dose, followed by $2 \mathrm{mg} \mathrm{kg}^{-1}$ weekly). Trastuzumab was combined with docetaxel $75 \mathrm{mg} \mathrm{m}^{-2}$ every 3 weeks in 42 patients, including 34 who were treated in a phase II multi-institutional trial (Montemurro et al, 2004a), and vinorelbine $30 \mathrm{mg} \mathrm{m}^{-2}$ on days 1 and 8 every 3 weeks for the remaining 12 patients. For both docetaxel and vinorelbine, the treatment was planned to be given for a maximum of six cycles with concomitant weekly Trastuzumab. Patients showing tumour response or stabilisation upon the completion of chemotherapy continued weekly Trastuzumab until disease progression or unacceptable toxicity.

Tumour response was classified according to WHO criteria (CR: complete remission, PR: partial remission, SD: stable disease, PD: progressive disease) (Miller et al, 1981). Response rate (RR, CR plus PR) is reported together with its $95 \%$ confidence interval (95\% CI), as calculated by the Wilson's method (Wilson, 1927). The Kaplan-Meier product limit method was used to determine: (1) time to progression (TTP), which was calculated from the date of the first dose of docetaxel and Trastuzumab and the date of the first documented tumour progression or death in the absence of tumour progression; (2) overall survival (OS), which was calculated from the date of the first dose of Trastuzumab to the date of documented death of the patients. Alive patients were censored at the date of most recent follow-up visit.

Statistical analysis The concordance among IHC assay methods was studied by the $\mathrm{K}$ statistics. The difference in RR between subgroups of patients was analysed by Fisher's Exact Test. Differences in TTP and OS between different subgroups of patients were analysed by the log-rank test. The effect of BiotHER positivity on RR was studied by logistic regression analysis including potential covariates. Results are reported as odds ratio (OR) with 95\% CI. Similarly, the effect of BiotHER positivity on TTP and OS was studied in a Cox's Proportional Hazards model including potential covariates. Results are reported as hazard ratio (HR) with 95\% CI. The $P$-values were calculated by means of the Wald statistic and considered significant if $\leqslant 0.05$. The statistical analysis was performed using the SPSS-PC software, version 11.5 (SPSS, Chicago, IL, USA).

\section{RESULTS}

\section{Definition of HER-2 expression by immunohistochemistry}

HER-2 protein receptor overexpression was located on membranes of BT474 breast cancer cells with all the mAbs tested, that is, Herceptest, TAB250 and BiotHER (Figure 2A). The quality control analysis demonstrated that conjugation process with biotin had a reproducibility of $100 \%$, while the immunoreactivity was lost after 3 months from biotinylation. Staining with BiotHER was invariably detected on the cell membrane, while cytoplasmic staining (Figure 2B) or staining of stroma and of normal residual epithelial cells was never observed (Figure 2C, D). Fluorescence in situ hybridisation analysis of the 164 specimens from primary breast cancers showed HER-2 gene amplification in 42 cases $(26 \%)$. The only 21 specimens that stained positively by BiotHER had HER-2 gene amplification (Figure 2E, F arrows). Concordance of BiotHER with Herceptest and TAB250 are summarised in Table 1a and b. MAb 4D5 was studied in 24 of the 164 breast carcinomas. Of 14 cases with HER-2 amplification, nine were positive with both BiotHER and 4D5, whereas two were negative with both tests, and in three cases 4D5 alone was positive. The 10 nonamplified cases studied were negative for both $4 \mathrm{D} 5$ and BiotHER staining ( $K$ for overall concordance $=0.75$, $P<0.01)$.

None of the other specimens from different cancers (see Materials and Methods) showed HER-2 gene amplification or BiotHER staining, except for two cases of colon cancer. Both cases were HER-2 gene amplified, BiotHER positive and TAB250 positive. Herceptest was positive in only one of these cases and negative in the other. 

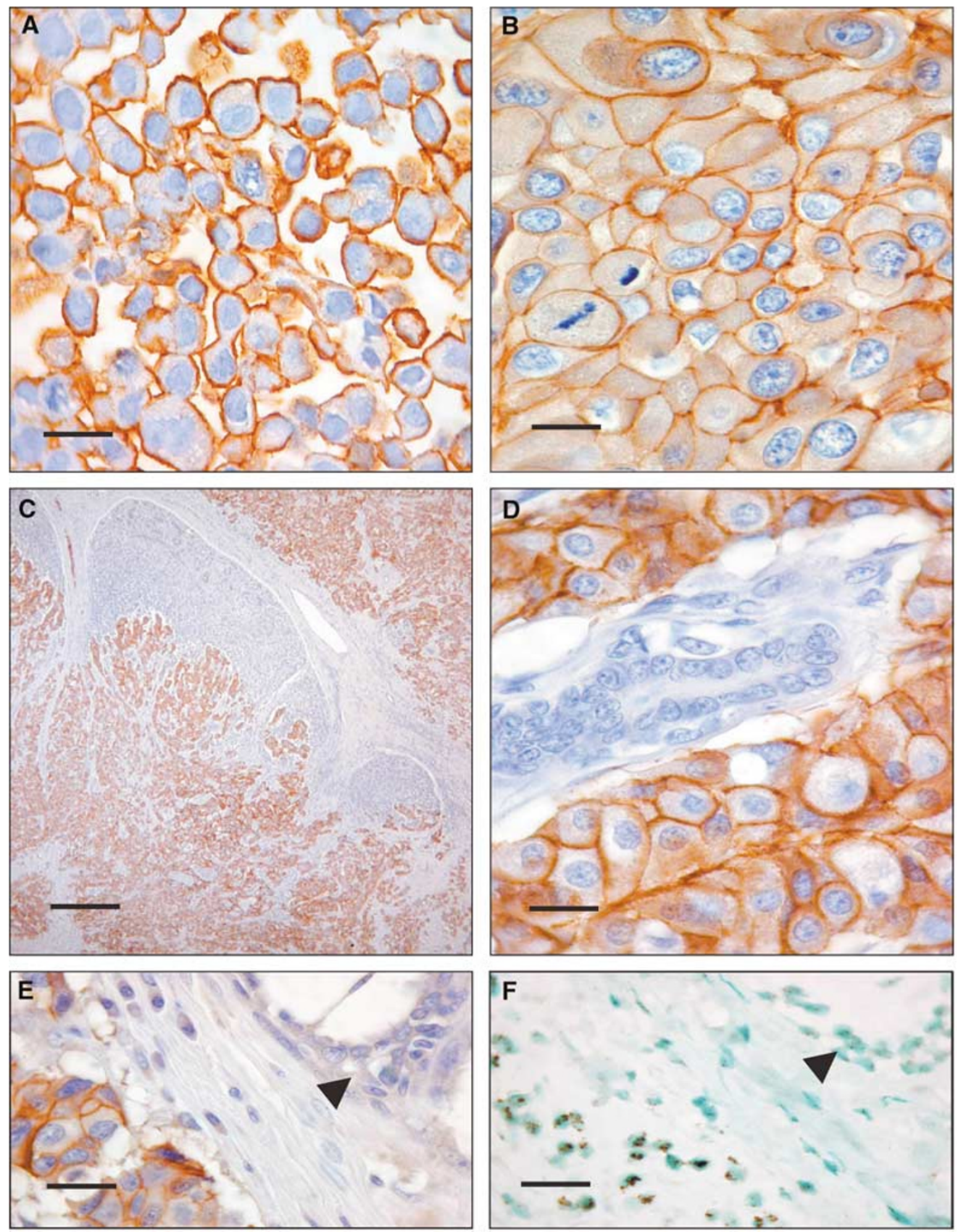

Figure 2 BiotHER immunostaining is restricted to membranes of $(\mathbf{A})$ BT474 cells and of (B) cancer cells in paraffin-embedded tumour samples; (C) no background or $(\mathbf{D})$ normal cell staining is observed. (E) BiotHER stains amplified tumour cells (F) (CISH; large gene copy cluster/nucleus), and (E, $\mathbf{F}$ arrows) is negative in normal nonamplified residual cells (two signals/nucleus).

Table I Concordance of BiotHER with (a) HercepTest, (b) TAB250

\begin{tabular}{lrcc}
\hline BiotHER score & $\mathbf{0 - 1}$ & $\mathbf{2 - 3}$ & Total No. of specimens \\
\hline (a) & 85 & 58 & 143 \\
$0-1$ & 1 & 20 & 21 \\
$2-3$ & & & \\
(b) & 109 & 34 & 143 \\
$0-1$ & 0 & 21 & 21 \\
$2-3$ & & & \\
\hline
\end{tabular}

Note: $K=0.45, P<0.01$.

\section{BiotHER immunostaining and clinical outcome in patients receiving Trastuzumab for advanced breast cancer}

Of the 54 tumour specimens from patients selected for the clinical outcome analysis, BiotHER was negative (score 0 or $1+$ ) in 27 cases and positive (score $2+$ or $3+$ ) in the remaining 27 cases $(50 \%)$. The original tissue specimens of this case series derived from different laboratories and were probably prepared using different fixation methods. Seven of these specimens showed unspecific immunostaining (cytoplasm and/or background staining and no clear membrane staining) with BiotHER and were thus ultimately considered negative. 
At the time of this analysis, 36 patients have progressed and 22 patients have died. The median follow-up for patients who were still alive at the last follow-up contact was 22 months (range 4-58 months). Overall, the combination of chemotherapy plus Trastuzumab produced a $74 \%$ RR (95\% CI $61-84 \%)$. The Kaplan-Meier estimates of median TTP for the overall population was 11 months (95\% CI 8-14 months, Figure 3). The median overall survival was not reached (Figure 4). The 2-year survival was 55\% (95\% CI 39 $71 \%)$.

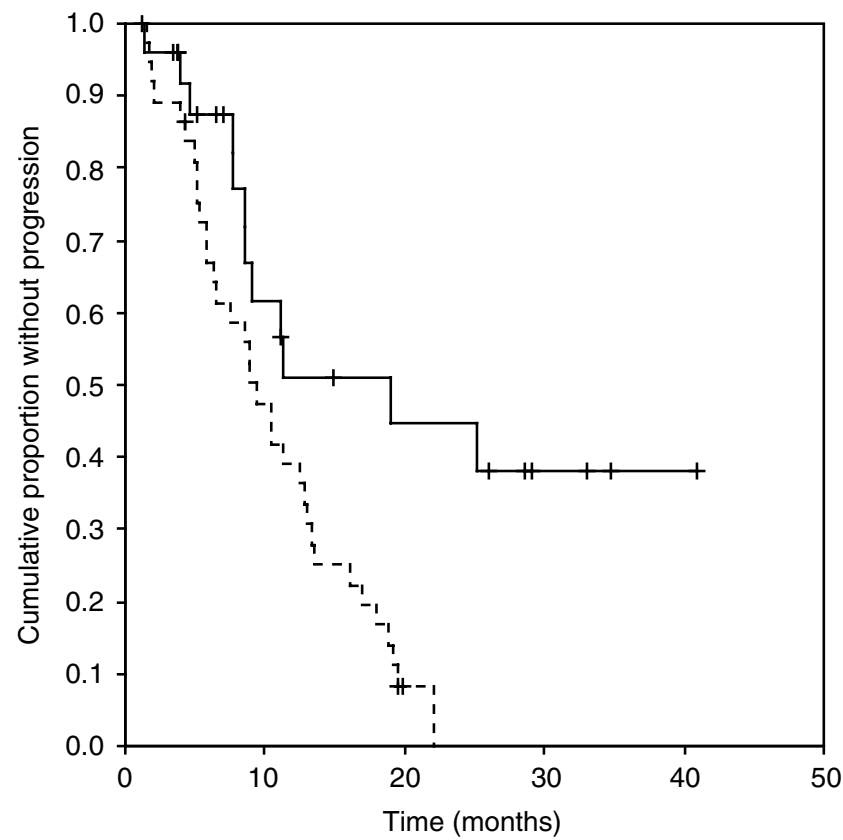

Figure 3 Kaplan-Meier estimates of time to progression (TTP) according to BiotHER status. The solid line represents patients with BiotHER-positive tumours and the dashed line patients with BiotHERnegative tumours.

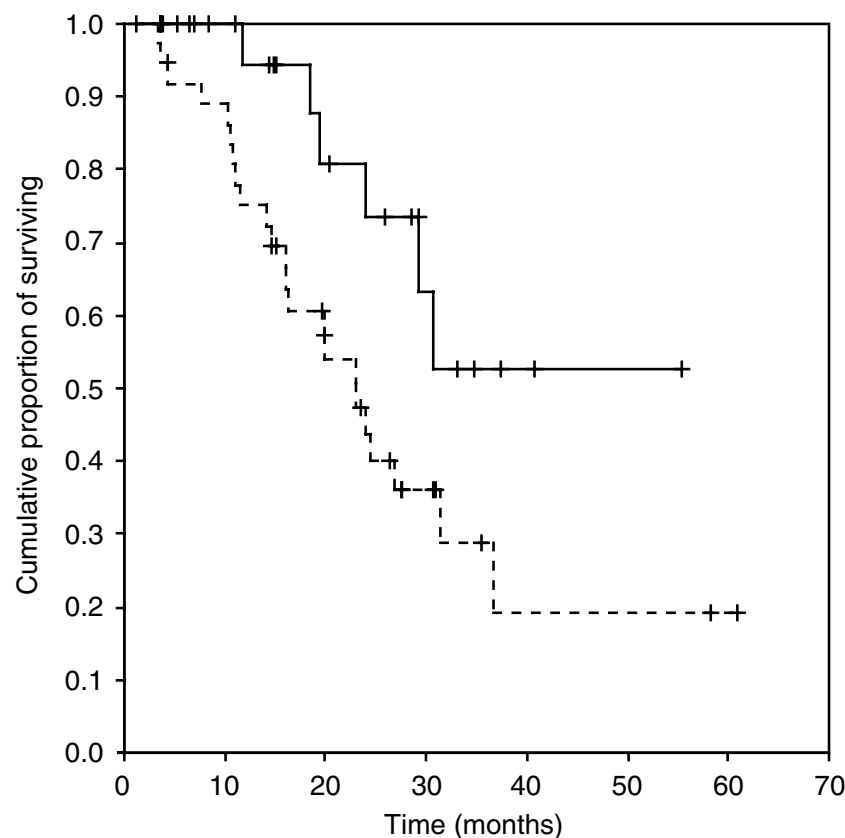

Figure 4 Kaplan-Meier estimates of overall survival (OS) according to BiotHER status. The solid line represents patients with BiotHER-positive tumours and the dashed line patients with BiotHER-negative tumours.
Table 2 Patients' demographics according to BiotHER status

\begin{tabular}{lcc}
\hline & BiotHER- & BiotHER+ \\
\cline { 2 - 3 } Characteristic & $\mathbf{N}=\mathbf{2 7}$ & $\mathbf{N}=\mathbf{2 7}$ \\
\hline Median age in years (range) & $55(35-75)$ & $50(36-76)$ \\
Median DFS in months (range) & $21(0-134)$ & $24(0-1 \mid 9)$ \\
Median number of metastatic sites (range) & $2(1-4)$ & $2(1-3)$ \\
Patients with single metastatic sites & $8(30 \%)$ & $11(41 \%)$ \\
Visceral involvement (liver+lung) & $19(70 \%)$ & $22(81 \%)$ \\
Liver involvement & $14(52 \%)$ & $15(56 \%)$ \\
Prior chemotherapy for metastatic disease & $18(67 \%)$ & $23(85 \%)$ \\
Prior adjuvant/neoadjuvant chemotherapy & $15(56 \%)$ & $18(67 \%)$ \\
Prior exposure to antracyclines & $18(67 \%)$ & $16(59 \%)$ \\
\hline
\end{tabular}

$\mathrm{N}=$ number; $\mathrm{DFS}=$ disease-free survival from initial diagnosis to metastatic progression

Table 3 Response to Trastuzumab-based therapy according to BiotHER status

\begin{tabular}{lcccc}
\hline Response & BiotHER pos. & Rate (\%) & BiotHER neg. & Rate (\%) \\
\hline CR+PR & 24 & $89^{b}$ & 16 & 59 \\
CR & 2 & 7 & 4 & 15 \\
PR & 22 & 81 & 12 & 44 \\
SD & 2 & 7 & 9 & 33 \\
PD & 1 & 4 & 2 & 7 \\
\hline
\end{tabular}

${ }^{\mathrm{a} B e c a u s e}$ of rounding, the sum of percentages is not always $=100 .{ }^{\mathrm{b}}$ Fisher's exact test for the difference in the rate of responders $(C R+P R), P=0.03$. $C R=$ complete remission; $\mathrm{PR}=$ partial remission; $\mathrm{SD}=$ stable disease $; \mathrm{PD}=$ progressive disease.

None of the differences seen in the distribution of clinical characteristics between patients with BiotHER-positive and negative tumours approached statistical significance (Table 2). The RR was significantly higher in BiotHER-positive patients (89\%, 95\% CI 72-96\%), compared to BiotHER-negative patients (59\%, 95\% CI 41-75\%) (Table 3).

BiotHER positivity was associated with longer median TTP (19 vs 9 months for patients with BiotHER-positive and -negative tumours, respectively, $P=0.03$ ) and median OS (not reached $v s 23$ months for BiotHER-positive and -negative tumours, respectively, $P=0.04$ ) (Figures 3 and 4 ).

The effect of BiotHER positivity on RR, TTP and OS was studied in multivariate analysis models including other variables, which were found to be significantly associated with the outcomes in the univariate analysis (data not shown). BiotHER positivity was independently associated with increased likelihood of tumour response (Table 4). Of the other potential covariates, only prior exposure to chemotherapy for metastatic disease was independently associated with reduced response rate. Survival analysis showed that BiotHER status was the only independent predictor for both OS and PFS. BiotHER positivity was associated with reduced risk of death (HR 0.391, 95\% CI 0.152-1.003, $P=0.05$ ) and tumour progression (HR $0.04,95 \%$ CI $0.213-0.912$, $P=0.03)$.

\section{DISCUSSION}

In the present work, we devised a simple strategy, based on the biotinylation of Trastuzumab (BiotHER), which allows the use of the anti-HER-2 humanised Ab for IHC. Thus, BiotHER can be used as a primary $\mathrm{Ab}$ when evaluating the availability of Trastuzumabspecific binding sites in breast tumour tissue sections. We 
Table 4 Logistic regression analysis of tumour response

\begin{tabular}{|c|c|c|c|c|c|c|}
\hline \multirow[b]{2}{*}{ Variable } & \multicolumn{3}{|c|}{ Univariate } & \multicolumn{3}{|c|}{ Multivariate } \\
\hline & OR & $95 \% \mathrm{Cl}$ & $P$ & OR & $95 \% \mathrm{Cl}$ & $P$ \\
\hline $\begin{array}{l}\text { BiotHER } \\
\text { positivity }\end{array}$ & 5.500 & $1.323-22.862$ & 0.04 & 4.589 & $1.042-20.207$ & 0.04 \\
\hline $\begin{array}{l}\text { Prior } \\
\text { chemotherapy } \\
\text { for metastases }\end{array}$ & 0.176 & $0.045-0.688$ & 0.01 & 0.216 & $0.052-0.906$ & 0.04 \\
\hline
\end{tabular}

$\mathrm{OR}=$ odds ratio; $\mathrm{Cl}=$ confidence interval.

demonstrated that BiotHER immunoreactivity was restricted to tumours with HER-2 gene amplification. In addition, BiotHER positivity was a strong predictor of clinical outcome in patients with advanced breast cancer treated with Trastuzumab and chemotherapy.

Trastuzumab is a human-murine chimeric $\mathrm{mAb}$, which retains only the small antigen-binding fragment of the murine 4D5 immunoglobulin (Carter et al, 1992) that specifically recognises the ECD of HER-2. In clinical trials, to screen patients for enrolment, immunostaining with $4 \mathrm{D} 5 \mathrm{mAb}$ was used but only in combination with other Abs (Mass et al, 2001; Slamon et al, 2001; Bookman et al, 2003) because of concerns that use of 4D5 mAb alone might underestimate HER-2 detection in formalin-fixed and paraffinembedded tissues. The fact that only a subset of FISH-amplified tumours scored positively by $4 \mathrm{D} 5$, as well as by BiotHER in the present study, may be explained by the shedding of ECD activated by proteolitic processes. In fact, it has been shown that $22.4 \%$ of breast cancer tissues express only the p95 $\mathrm{NH}_{2}$-truncated form of HER-2 (Christianson et al, 1998), owing to shedding of ECD. The possible truncation of HER-2 with loss of ECD prompted us to use Trastuzumab as the primary $\mathrm{Ab}$ in IHC procedures, in order to select patients whose tumour cells maintain the target epitope. In addition, 4D5 is not commercially available. HER-2 diagnostic testing recommendations (Bilous et al, 2003), proposed by different nations, do not consider $4 \mathrm{D} 5 \mathrm{mAb}$ as an IHC marker and, to our knowledge, no work has been published on the correlation between 4D5 immunostaining and HER-2 gene amplification by FISH analysis in tissue specimens. We decided upon biotinylation of Trastuzumab to avoid incubation with a secondary anti-human IgG, which could create problems of background staining. In addition to reducing the IHC procedure to a single step (no secondary antibody needed), BiotHER immunoreaction does not require antigen retrieval. In properlyfixed tissue samples, we observed that: (i) the IHC reaction with BiotHER was limited to the plasma membrane of tumour cells; (ii) no cytoplasmic staining was observed and (iii) normal epithelial or stromal cells did not react whatsoever. However, in the series of patients treated with Trastuzumab, we noted suboptimal (i.e., cytoplasmic and background) BiotHER staining in seven cases (three tumours were nonresponsive and four responded to Trastuzumab treatment) received from external laboratories. These data confirm the need for optimal tissue processing as a prerequisite for reliable HER-2 IHC staining (Bilous et al, 2003). Comparison of the reactivity of BiotHER with that of other commercially available Abs such as Herceptest and TAB250 demonstrated a low concordance.

Not surprisingly, we observed instead an $87.5 \%$ agreement of the results obtained with BiotHER and mAb 4D5; while $12.5 \%$ of IHC reactivity with mAb 4D5 was not confirmed with BiotHER. The reason for this minor discrepancy is not clear since Trastuzumab has a high affinity for the HER-2 ECD $\left(K_{\mathrm{d}}=5 \times 10^{-9} \mathrm{M}\right)$ although we cannot exclude that a slight structural variation of the 4D5 Fab occurred after humanisation. In fact, the crystal structure of $\mathrm{mAb}$ $4 \mathrm{D} 5$ demonstrates that the complementarity determining region 3 , which determines the antibody-antigen interaction, is composed of six highly flexible hypervariable loops (Zhang et al, 1999).

When we calculated the specificity of BiotHER reactivity using the known HER-2 gene status of the tumour as the gold standard, we observed that none of the unamplified samples were BiotHERor 4D5-positive. In addition, differently from Herceptest and TAB250, all cases positive with BiotHER even those scored as $2+$ showed invariably high level of gene amplification by FISH analysis (50\% sensitivity) and all cases scored as $1+$ or 0 were nonamplified ( $100 \%$ specificity).

The second part of our study evaluated the clinical impact of the results of BiotHER IHC staining. For this purpose, predictivity of BiotHER results with respect to RR, TTP and OS was retrospectively studied in a series of 54 patients with HER-2 amplified advanced breast cancer who had received Trastuzumab plus chemotherapy. Patients whose tumours was BiotHER positive had superior RR, TTP and OS, compared with patients with BiotHERnegative tumours. BiotHER positivity was an independent predictor of clinical outcome in both the overall and the amplified population. We thus suggest that BiotHER identifies the subset of patients with HER-2 amplified tumours achieving the highest benefit from Trastuzumab-based therapy. Obviously, these results should be interpreted with caution and require prospective confirmation for two main reasons: (1) the analysis included a small number of subjects, (2) in the amplified population, the response rate in patients with BiotHER-negative tumours was $59 \%$, a rate that does not exclude a possible, even if less pronounced, benefit from the addition of Trastuzumab to chemotherapy also in this subset of patients. On the other hand, our findings provide a possible explanation for the low activity rate of single agent Trastuzumab in patients selected on the basis of conventional IHC testing and FISH. As stated before, the ECD of HER-2, which represents the accessible target for immunotherapy with Trastuzumab, can be shed (Hayes et al, 2001) and splice variants of HER-2 with deletion in ECD exons can occur (Christianson et al, 1998; Kwong and Hung, 1998; Molina et al, 2002). Another explanation to the response of patients with negative BiotHER immunoreaction is that mechanisms of HER-2 shedding might change under Trastuzumab therapy and chemotherapy, with less of the HER-2 protein fragment being released to the serum, which would make the c-erbB-2-positive tumour cells a better target for anti-HER-2 antibody treatment (Luftner et al, 1999; Molina et al, 2001).

Both FISH testing and Abs that are directed against the intracellular domain, such as Herceptest and CB11, or against undefined ECD epitopes, such as TAB250, do not account for the possible absence of the Trastuzumab epitope on the cell surface.

In conclusion, our results suggest that, in patients with breast cancer and HER-2 gene amplification, the use of BiotHER as primary $\mathrm{Ab}$ in IHC procedures has the potential to further improve the definition of the target population for Trastuzumab-based treatment. Furthermore, we provide the first evidence that mAbs humanised for use in therapy (an increasingly popular approach) can be used in IHC, simultaneously revealing target availability and forecasting clinical efficacy.

\section{ACKNOWLEDGEMENTS}

This work was supported by grants from the Ministry for Universities, Instruction and Research (MIUR), Rome, Italy; MURST (ex 60\%), Rome, Italy; the Compagnia di San Paolo/ FIRMS, Torino, Italy. We are grateful to Laura Casorzo, Rosanna Lupo and Patrizia Gugliotta for technical assistance and Dr Enza Ferrero (Laboratory of Immunogenetics, Department of Genetics, Biology and Biochemistry) for suggestions and the careful revision of the manuscript. 


\section{REFERENCES}

Baselga J, Norton L, Albanell J, Kim YM, Mendelsohn J (1998) Recombinant humanized anti-HER-2 antibody (Herceptin) enhances the antitumour activity of paclitaxel and doxorubicin against HER2/neu overexpressing human breast cancer xenografts. Cancer Res 58: 2825-2831

Bilous M, Dowsett M, Hanna W, Isola J, Lebeau A, Moreno A, PenaultLlorca F, Ruschoff J, Tomasic G, van de Vijver M (2003) Current perspectives on HER2 testing: a review of national testing guidelines. Mod Pathol 16: $173-182$

Bookman MA, Darcy KM, Clarke-Pearson D, Boothby RA, Horowitz IR (2003) Evaluation of monoclonal humanized anti-HER2 antibody, trastuzumab, in patients with recurrent or refractory ovarian or primary peritoneal carcinoma with overexpression of HER2: a phase II trial of the Gynecologic Oncology Group. J Clin Oncol 21: 283-290

Carter P, Presta L, Gorman CM, Ridgway JB, Henner D, Wong WL, Rowland AM, Kotts C, Carver ME, Shepard HM (1992) Humanization of an anti-p185HER2 antibody for human cancer therapy. Proc Natl Acad Sci USA 89: $4285-4289$

Ceccarelli C, Santini D, Gamberini M, Taffurelli M, Chieco P, Piana S, Pileri S, Marrano D (1999) Immunohistochemical expression of internal and external ErbB-2 domains in invasive breast cancer. Breast Cancer Res Treat 58: $107-114$

Cho HS, Mason K, Ramyar KX, Stanley AM, Gabelli SB, Denney Jr DW, Leahy DJ (2003) Structure of the extracellular region of HER2 alone and in complex with the Herceptin Fab. Nature 421: 756-760

Christianson TA, Doherty JK, Lin YJ, Ramsey EE, Holmes R, Keenan EJ, Clinton GM (1998) $\mathrm{NH}_{2}$-terminally truncated HER-2/neu protein: relationship with shedding of the extracellular domain and with prognostic factors in breast cancer. Cancer Res 58: 5123-5129

Cobleigh MA, Vogel CL, Tripathy D, Robert NJ, Scholl S, Fehrenbacher L, Wolter JM, Paton V, Shak S, Lieberman G, Slamon DJ (1999) Multinational study of the efficacy and safety of humanized anti-HER2 monoclonal antibody in women who have HER2-overexpressing metastatic breast cancer that has progressed after chemotherapy for metastatic disease. J Clin Oncol 17: 2639-2648

Codony-Servat J, Albanell J, Lopez-Talavera JC, Arribas J, Baselga J (1999) Cleavage of the HER2 ectodomain is a pervanadate activable process that is inhibited by the tissue inhibitor of metalloproteases-1 in breast cancer cells. Cancer Res 59: 1196-1201

Corbett IP, Henry JA, Angus B, Watchorn CJ, Wilkinson L, Hennessy C, Gullick WJ, Tuzi NL, May FE, Westley BR, Horne CHW (1990) NCLCB11, a new monoclonal antibody recognizing the internal domain of the c-erbB-2 oncogene protein effective for use on formalin-fixed, paraffinembedded tissue. J Pathol 161: 15-25

Elkin EB, Weinstein MC, Winer EP, Kuntz KM, Schnitt SJ, Weeks JC (2004) HER-2 testing and trastuzumab therapy for metastatic breast cancer: a cost-effectiveness analysis. J Clin Oncol 22: 854-863

Hayes DF, Yamauchi H, Broadwater G, Cirrincione CT, Rodrigue SP, Berry DA, Younger J, Panasci LL, Millard F, Duggan DB, Norton L, Henderson IC (2001) Circulating HER-2/erbB-2/c-neu (HER-2) extracellular domain as a prognostic factor in patients with metastatic breast cancer: Cancer and Leukemia Group B Study 8662. Clin Cancer Res 7: 2703-2711

Kwong KY, Hung MC (1998) A novel splice variant of HER2 with increased transformation activity. Mol Carcinog 23: 62-68

Luftner D, Schnabel S, Possinger K (1999) c-erbB-2 in serum of patients receiving fractionated paclitaxel chemotherapy. Int J Biol Markers 14: $55-59$
Mass RD, Press M, Anderson S, Murphy M, Slahon D (2001) Improved survival benefit from Herceptin (trastuzumab) in patients selected by fluorescence in situ hybridization (FISH). Proc Am Soc Clin Oncol 20: 22a (abstr 85)

Miller AB, Hoogstraten B, Staquet M, Winkler A (1981) Reporting results of cancer treatment. Cancer 47: $207-214$

Molina MA, Codony-Servat J, Albanell J, Rojo F, Arribas J, Baselga J (2001) Trastuzumab (herceptin), a humanized anti-Her2 receptor monoclonal antibody, inhibits basal and activated Her2 ectodomain cleavage in breast cancer cells. Cancer Res 61: $4744-4749$

Molina MA, Saez R, Ramsey EE, Garcia-Barchino MJ, Rojo F, Evans AJ, Albanell J, Keenan EJ, Lluch A, Garcia-Conde J, Baselga J, Clinton GM (2002) $\mathrm{NH}(2)$-terminal truncated HER-2 protein but not full-length receptor is associated with nodal metastasis in human breast cancer. Clin Cancer Res 8: $347-353$

Montemurro F, Choa G, Faggiuolo R, Donadio M, Minischetti M, Durando A, Capaldi A, Vietti-Ramus G, Alabiso O, Aglietta M (2004a) A phase II study of three-weekly docetaxel and weekly Trastuzumab in HER2overexpressing advanced breast cancer. Oncology 66: 38-45

Montemurro F, Valabrega G, Aglietta M (2004b) Trastuzumab-based combination therapy for breast cancer. Exp Opin Pharmacother 5: 81 - 96

Pauletti G, Godolphin W, Press MF, Slamon DJ (1996) Detection and quantitation of HER-2/neu gene amplification in human breast cancer archival material using fluorescence in situ hybridization. Oncogene 13: $63-72$

Sapino A, Coccorullo Z, Cassoni P, Ghisolfi G, Gugliotta P, Bongiovanni M, Arisio R, Crafa P, Bussolati G (2003) Which breast carcinomas need HER-2/neu gene study after immunohistochemical analysis? Results of combined use of antibodies against different c-erbB2 protein domains. Histopathology 43: $354-362$

Slamon DJ, Clark GM, Wong SG, Levin WJ, Ullrich A, McGuire WL (1987) Human breast cancer: correlation of relapse and survival with amplification of the HER-2/neu oncogene. Science 235: 177-182

Slamon DJ, Godolphin W, Jones LA, Holt JA, Wong SG, Keith DE, Levin WJ, Stuart SG, Udove J, Ullrich A, Press MF (1989) Studies of the HER-2/ neu proto-oncogene in human breast and ovarian cancer. Science 244: $707-712$

Slamon DJ, Leyland-Jones B, Shak S, Fuchs H, Paton V, Bajamonde A, Fleming T, Eiermann W, Wolter J, Pegram M, Baselga J, Norton L (2001) Use of chemotherapy plus a monoclonal antibody against HER2 for metastatic breast cancer that overexpresses HER2. $N$ Engl J Med 344: $783-792$

Tokuda Y, Ohnishi Y, Shimamura K, Iwasawa M, Yoshimura M, Ueyama Y, Tamaoki N, Tajima T, Mitomi T (1996) In vitro and in vivo anti-tumour effects of a humanised monoclonal antibody against c-erbB-2 product. $\mathrm{Br}$ J Cancer 73: $1362-1365$

Vogel CL, Cobleigh MA, Tripathy D, Gutheil JC, Harris LN, Fehrenbacher L, Slamon DJ, Murphy M, Novotny WF, Burchmore M, Shak S, Stewart SJ, Press M (2002) Efficacy and safety of trastuzumab as a single agent in first-line treatment of HER2-overexpressing metastatic breast cancer. J Clin Oncol 20: $719-726$

Wilson EB (1927) Probable inference, the law of succession, and statistical inference. J Am Statist Assoc 22: 209-212

Zhang H, Wang Q, Montone KT, Peavey JE, Drebin JA, Greene MI, Murali R (1999) Shared antigenic epitopes and pathobiological functions of anti-p185(her2/neu) monoclonal antibodies. Exp Mol Pathol 67: 15-25 